\title{
Time-resolved macromolecular crystallography: no longer a niche pursuit?
}

\author{
Arwen R Pearson \\ University of Hamburg, Hamburg, Germany; \\ arwen.pearson@cfel.de
}

The advances over the last decade in ultrabright X-ray sources have reinvigorated interest in time-resolved structural biology. Importantly, many of the time-scales of interest to structural biology are in fact accessible using serial crystallographic approaches at synchrotrons. Therefore, although the experiments themselves remain challenging, a number of new beamlines and endstations are coming online at synchrotron sources to serve this growing community. This has been complemented by increasing exchange between synchrotron and XFEL researchers, sharing methods, data processing tools and even supporting experiments that make use of both types of light source.

To help structural biologists take full advantage of these new resources, considerable effort is being put in to help interested researchers to optimise their sample in terms of crystal quantity, size and quality, as well as determine how to trigger the reaction of interest as uniformly as possible in each crystal. Key here is that optimising the sample and ensuring that sufficient data are collected to provide clear electron density maps at each time point requires both regular beamtime access and rapid feedback during the experiment.

The T-REXX endstation on beamline P14 at PETRA III has been built to address these challenges by a collaborative team from EMBL, Universität Hamburg and the Max Planck Institute for the Structure and Dynamics of Matter. It is dedicated to serial crystallographic data collection, and has an open design that can accommodate a range of serial sample mounts as well as different reaction initiation methods. A number of tools have been developed by the T-REXX collaboration for sample preparation and mounting, as well as protocols for sample and data collection optimisation. Rapid feedback on hit rate and resolution is presented in the controls GUI, and automatic processing pipelines deliver first maps a few hours after data collection is complete.

In this presentation I will present the current state of T-REXX, including recent results, and some of the tools we have developed to facilitate sample optimsation and mounting, reaction initiation and data processing. I will also take a look at the wider field and highlight some of the remaining challenges and opportunities.

Keywords: time-resolved structural biology, mechanistic enzymology 